\title{
Identification of cases of sudden infant death syndrome from death certificates
}

\author{
JEAN W KEELING, ${ }^{1}$ JEAN GOLDING ${ }^{2}$ AND BETTINE SUTTON ${ }^{3}$ \\ From the John Radcliffe Hospital, ${ }^{1}$ Oxford, Department of Child Health, ${ }^{2}$ University of Bristol, and Unit of \\ Clinical Epidemiology, ${ }^{3}$ University of Oxford
}

SUMMARY A method is proposed for identification of cases of sudden infant death syndrome (SIDS) from information available on death certificates. Deaths at ages between 7 days and 2 years, referred to a coroner, having certain specified causes of death codes, identified 160 of 169 cases of SIDS confirmed as such by a pathologist. The sensitivity of the method was $94 \%$ and the specificity was $97 \%$.

The application of Beckwith's definition' ${ }^{1}$ of sudden infant death syndrome (SIDS): "The sudden death of any infant or young child which is unexpected by history and in whom a thorough necropsy examination fails to demonstrate an adequate cause of death" is a process of elimination rather than the identification of positive criteria. In a population where a single pathologist is responsible for all necropsy examinations, the classification will be relatively consistent, especially as this conclusion is drawn for each case after the completion of all investigations. When more than one pathologist is involved, however, there is likely to be lack of concordance because of the differences in interpretation of pathological abnormalities observed and their relation to death. This makes epidemiological studies of SIDS particularly difficult; for an epidemiologist, it is obviously important to ascertain all cases that occur in a defined population, which is why ascertainment based on death certificates is attractive.

Until 1971 it was not possible to register infant deaths as sudden and unexpected in Britain. Since then the option has probably not been used as frequently as it should be: ${ }^{2}$ both pathologists and coroners seem reluctant to admit that a particular death is unexplained. By law all deaths have to be registered, but there is no penalty for inaccuracy. Considerable disparity has been demonstrated between registered causes of death and pathological findings in neonatal deaths. ${ }^{3}$ In Seattle, Peterson and colleagues $^{4}$ tried to identify cases of SIDS from death certificates among the 0-2 year age group and then examined their findings against detailed pathological investigations which had been undertaken. They found that by restricting their interest to babies dying between 1 and 4 months of age and excluding deaths due to malformation or birth related conditions (coded 740-799) they identified 246 deaths. Only $175(71 \%$ of these) were cases of SIDS, and their restrictions had omitted a further 33 SIDS. Clearly, further discrimination was necessary to achieve useful identification from death certificates. In $-\vec{C}$ England and Wales, Gardner ${ }^{2}$ compared necropsy findings on deaths in Sheffield with informationg recorded on death certificates. She found a serious lack of concordance, one third of those deaths registered as sudden infant deaths actually being classified otherwise by the reviewing pathologist.

The aim of the present study was to look at deaths of infants in a defined population, certified by many different coroners and medical practitioners, to see whether a more accurate method of assessing the number of cases of SIDS could be made from death registrations.

\section{Materials and methods}

Copies of the birth certificates, death certificates, and details of hospital admissions of all residents of the Oxford region are on the files of the Oxford Record Linkage Study. ${ }^{5}$ For this project, we reviewed all deaths occurring between 1971 and 1975 to children aged 7 days to 1 year 364 days, resident in Oxfordshire and West Berkshire at the time of death. The area studied is largely of rural composition but includes the cities of Reading and Oxford. The population in 1971 was 856000 and there were 65000 births to residents in the area during the five years 1971 to 1975 .

In England and Wales, a death must be referred to a coroner either when the medical practitioner has 
not seen the patient within 14 days before death or when the antecedent history of illness is insufficient to enable him to issue a certificate. The coroner may then arrange for necropsy examination and issue a death certificate based on those findings. Clinical information on cases referred to a coroner was difficult to obtain, except for those deaths that occurred in hospital. There was scant clinical information available from coroner's records. The pathologists who had carried out the necropsy examinations were a much more fruitful source of information and were happy, with the consent of the coroner, to supply copies of the postmortem reports which often had relevant clinical details appended.

When necropsy examination had been carried out, a copy of the postmortem report was requested and the histology was examined and assessed by the first author. Histological abnormalities present were graded as (A) no pathological abnormality, (B) minor pathological abnormality, but no indication of a specific disease process, (C) evidence of disease but thought not to be severe enough to explain death, and (D) disease of such severity as to be considered a cause of death. On the basis of the collected information, deaths were divided into two groups: "explained" (D) and "unexplained" (A-C), and defined as "sudden unexpected" if they had been referred to a coroner.

After classifying the deaths in this way, the classification was then compared with the registered causes of death as coded on the death certificate.

\section{Results}

There were 456 deaths to children aged from 7 days to 1 year 364 days during the five year period. Of these, $45(10 \%)$ had occurred outside the area, even though the child was still a resident of the area. Table 1 shows the primary registered cause of death grouped according to the Eighth Revision of the International Classification of Diseases in three age groups: 7-27 days (late neonatal deaths), 28 days-51 weeks (postneonatal infant deaths) and 52 weeks-103 weeks (second year infant deaths). Congenital anomaly was the most frequent cause of late neonatal death, accounting for $58 \%$ of cases, followed by diseases associated with problems occurring in the perinatal period $(20 \%)$. Among postneonatal and second year deaths, about a quarter were ascribed to congenital anomalies and a further quarter were registered as respiratory disorders. Only 45 deaths were coded as sudden infant death syndrome.

Of the 456 deaths, $220(48 \%)$ had been referred to a coroner, but the proportions were different in the late neonatal period compared with the other age groups (table 1). Among late neonatal deaths, $10 \%$ were referred to a coroner, but the proportions for postneonatal and second year deaths were $60 \%$ and $53 \%$ respectively.

All deaths coded as due to accident, poisoning or violence had been referred to a coroner as had the 49 coded as "sudden infant death syndrome" (Code 795). All diagnostic groups containing more than 10 deaths included at least one where a coroner had been involved. This happened most frequently for respiratory disorders $(71,68 \%)$ and "infective and parasitic disease" $(17,49 \%)$.

The 220 deaths referred to a coroner included 55 which had sufficiently severe pathological abnormalities to explain death (27 of these being non natural deaths).

Table 1 Deaths by primary cause of death (ICD 8th rev)

\begin{tabular}{|c|c|c|c|c|}
\hline \multirow[b]{2}{*}{ Code } & \multirow[b]{2}{*}{ Primary cause of death } & \multicolumn{3}{|l|}{ Died } \\
\hline & & $7-27$ days & 28 days -51 weeks & 52-103 weeks \\
\hline \multirow{15}{*}{$\begin{array}{l}001-136 \\
140-239 \\
240-279 \\
280-289 \\
320-389 \\
390-458 \\
460-519 \\
520-577 \\
580-629 \\
710-738 \\
740-759 \\
760-779 \\
780-796 \\
\text { N800-N999 }\end{array}$} & Infective and parasitic disease & $5(0)$ & $25(15)^{*}$ & $5(2)$ \\
\hline & Benign and malignant neoplasms & 0 & $4(0)$ & $6(1)$ \\
\hline & Endocrine, nutritional and metabolic disease & $1(1)$ & $6(0)$ & 0 \\
\hline & Diseases of blood and blood-forming organs & 0 & $1(0)$ & $\mathbf{0}$ \\
\hline & Diseases of nervous system and sense organs & 0 & $9(0)$ & $6(3)$ \\
\hline & Diseases of circulatory system & $1(0)$ & 3 (1) & 0 \\
\hline & Diseases of respiratory system & 8(4) & $87(61)$ & $10(6)$ \\
\hline & Diseases of digestive system & $3(0)$ & $6(0)$ & $1(0)$ \\
\hline & Diseases of genitourinary system & 0 & $1(0)$ & 0 \\
\hline & Diseases of musculoskeletal system & $\mathbf{0}$ & 1 (0) & 0 \\
\hline & Congenital anomalies & $51(2)$ & 79 (13) & $16(3)$ \\
\hline & Certain causes of perinatal morbidity and mortality & 18(1) & 6 (1) & 0 \\
\hline & Symptoms and ill-defined conditions (included SIDS) & $\mathbf{0}$ & 49 (48) & $2(2)$ \\
\hline & Accidents, poisonings, and violence & 1(1) & $30(30)$ & 15(15) \\
\hline & All & 88(9) & $307(169)$ & $61(32)$ \\
\hline
\end{tabular}

*Cases referred to coroner in parentheses. 
In only 82 of the remaining 165 deaths referred to a coroner was histological material available for assessment. Although over half of these 82 cases had some histological abnormality (grade B) only a small number $(6 \%)$ had evidence of disease process within a particular system (grade C), and one third had no histological abnormality at all (grade A). None of these cases had any evidence of disease process sufficient to account for death. Of the group A cases, almost half were registered as sudden infant death syndrome (table 2), one was said to have died of inhalation of vomit, 11 were registered as respiratory disease, and two were said to have died as a result of congenital defect.

Table 2 Classification of pathological changes in cases of sudden infant death syndrome according to registered cause of death

\begin{tabular}{llrrrr}
\hline Code & Registered cause & $A$ & $B$ & $C$ & $A l l$ \\
\hline $008-9$ & Gastroenteritis & 0 & 4 & 2 & 6 \\
$422-519$ & Circulatory and respiratory & 11 & 25 & 2 & 38 \\
795 & Sudden unexpected death & 13 & 15 & 1 & 29 \\
933 & Inhalation of foreign body & 1 & 4 & 0 & 5 \\
& Remainder & 1 & 2 & 0 & 3 \\
& All & 26 & 50 & 5 & 81 \\
\hline
\end{tabular}

Although histological material was available on only half of the 165 deaths, the group without available histology had similar naked-eye descriptions of postmortem findings to those where we were able to examine the histology. It seems likely therefore that all should have been classified as sudden infant death syndrome.

When ICD codes of death certificate diagnoses in all deaths that appeared to be unexplained were re-examined, only the following groups were represented: 008-009 gastroenteritis, 422-519 circulatory and respiratory abnormalities, 795 sudden infant death syndrome, 933 ingestion of foreign body (including inhalation of vomit), and 994.7 asphyxia.

In order to determine whether any of the deaths that had not been referred to a coroner could have been examples of sudden infant death syndrome, all case notes were scrutinised. Out of the 236 deaths, there were two possible candidates: one, a "near-miss cot death" at 15 weeks died four weeks later after prolonged convulsions. The second baby died at home aged 4 weeks and was certified by a general practitioner without necropsy. In both cases the registered cause of death was respiratory infection. We added these two cases to the group of unexplained deaths (table 3 ):
Table 3 Deaths by referral to a coroner and age at death

\begin{tabular}{lllll}
\hline \multirow{2}{*}{$\begin{array}{l}\text { Referred to } \\
\text { coroner }\end{array}$} & \multicolumn{2}{l}{ Age at death } & \\
\cline { 2 - 5 } & $7-27$ days & 28 days-1 yr & $1-2$ yr & All \\
\hline Yes & $9(3)^{*}$ & $185(154)$ & $32(10)$ & $226(167)$ \\
No & $79(0)$ & $123(2)$ & $28(0)$ & $230(2)$ \\
All & $88(3)$ & $308(156)$ & $60(10)$ & $456(169)$ \\
\hline
\end{tabular}

*Numbers who satisfy the criteria for sudden infant death syndrome in parentheses.

An error in ascertainment might have been introduced by taking the age limits of 7 days and 103 weeks. We therefore examined the death certificates and other available information on deaths occurring in the first week of life and in children aged 2 to 4 years. There were 450 early neonatal deaths to parents resident in the area. Only two $(0.4 \%)$ could have been considered sudden and unexpected. Among the 113 children dying between 2 and 4 years of age, eight fulfilled the suggested criteria for ascertainment of sudden infant death syndrome outlined above: three of the eight deaths wereo considered to be explained when necropsy reports were scrutinised. Details of the remaining five couldo not be obtained (all had died outside the area).

\section{Discussion}

Between one third and one half of infant deathso occurring between 1 week and 1 year of age are, in this and most developed countries, considered to be unexplained at the present time. The majority of unexplained deaths occur between 6 and 24 weeks of age with a peak incidence at 12 weeks. ${ }^{78}$ The phrase "sudden infant death syndrome" (SIDS) or a synonym is currently used to identify this group of deaths, but this is not a disease, it is a conclusion arrived at by a process of elimination and exclusion. It cannot be reached without recourse to detailed necropsy examination including histological, microbiological, and biochemical investigations. ${ }^{9}$ Under this umbrella may be collected deaths from several disparate causes, ${ }^{10}$ many perhaps having a similar mode of dying. ${ }^{11}$

The purpose of this study was to try to devise a scheme so that examples of sudden infant death syndrome could be identified by examination of death certificates. The use of death certification as a starting point has as its main attraction the ability to relate the deaths to a particular population.

In this study, both clinical and pathological information was sought on each death in an attempt to sustain or refute the death certificate diagnosis. If we consider as "sudden" those deaths that have been 
referred to a coroner, then in this study we are likely to have omitted, at most, only two possible examples of sudden infant death syndrome from the 7 day to 2 year age group. If, however, we had used this as the sole criterion for selection we would have included 54 cases where death was actually found to have been explained. If we take only those deaths referred to a coroner which were coded on the death certificate within ICD groups 008-009 (gastroenteritis), 422-519 (circulatory and respiratory abnormalities), 795 (sudden infant death syndrome), 933 (ingestion of foreign body; especially inhalation of vomit), 994.7 (asphyxia), then this would exclude most of the explained deaths and leave us with 170 out of the original 456 deaths in the age group 7 days to 103 weeks (table 4). Of these, 10 would actually be explained, but another nine unexplained deaths would have been omitted, giving a similar number of false positives and false negatives. This gives a specificity of $97 \%$ and sensitivity of $94 \%$. In many epidemiological studies this would be considered an acceptable error rate.

Table 4 Case ascertainment based on details of postmortem and clinical history compared with identification from death certificates using rubrics 008-9, 422-519, 795, $933,944.7$ in deaths referred to a coroner (only deaths between 7 days and 2 years)

\begin{tabular}{lccc}
\hline \multirow{2}{*}{$\begin{array}{l}\text { Death certificate } \\
\text { assessment }\end{array}$} & \multicolumn{2}{l}{ Pathological assessment } \\
\cline { 2 - 4 } & SIDS & Non-SIDS & All \\
\hline SIDS & 160 & 10 & 170 \\
Non-SIDS & 9 & 277 & 286 \\
All & 169 & 287 & 456 \\
\hline
\end{tabular}

Nevertheless it must be remembered that we have defined our criteria from the observations made and then judged the efficiency of those criteria on the same observations. Clearly the method we have developed should be validated on other data sets.

\section{References}

${ }^{1}$ Beckwith JB. Observations on the pathological anatomy of the sudden infant death syndrome. In: Sudden infant death syndrome: Proceedings of the Second International Conference on Causes of Sudden Death in Infants, Bergman AB, Beckwith JB, Ray CG, eds. Seattle: University of Washington Press, 1970, 83.

${ }^{2}$ Gardner A. An attempt to identify cases of sudden infant death syndrome (cot death) from death certificates and hence determine the incidence. In: Studies in sudden infant deaths. Studies on Medical and Population Subjects No. 45. London: HMSO, 1982.

${ }^{3}$ Fedrick J, Butler NR. Accuracy of registered causes of neonatal deaths in 1958. Br J Prev Soc Med 1972; 26: 101-5.

${ }^{4}$ Peterson DR, Thompson DJ, Chinn NM. A method for assessing the geographic distribution and temporal trends of the sudden infant death syndrome in the United States from vital statistics data. Am J Epidemiol 1974; 100: 373-9.

${ }^{5}$ Acheson ED. Medical record linkage. London: Oxford University Press, 1967

${ }^{6}$ Baldwin JA. The Oxford Record Linkage Study as a medical information system. Proc Roy Soc Med 1972; 65: 237.

${ }^{7}$ Valdes-Dapena MA. Sudden and unexpected death in infancy: a review of the world literature 1954-66. Paediatrics 1967; 39: 123.

${ }^{8}$ Fedrick J. Sudden unexpected deaths in infants in the Oxford Record Linkage Area. Br J Prev Soc Med 1973; 27: 217-24.

${ }^{9}$ Keeling JW. Sudden infant death syndrome and non-accidental injury. In: Paediatric pathology, Berry CL, ed. 1981, 671-86.

${ }^{10}$ Anon. What kind of cot death. Br Med J 1978; 4: 671-2.

${ }^{11}$ Downing SE, Lee JC. Laryngeal chemosensitivity: a possible mechanism for sudden infant death. Pediatrics 1975; 55: 640-9. 